\title{
Increased corticomuscular coherence in idiopathic REM sleep behavior disorder
}

\section{Ki-Young Jung ${ }^{1}$ *, Jae-Hyun Cho ${ }^{2,3}$, Deokwon Ko ${ }^{1,4}$, Hung Youl Seok ${ }^{1}$, Ho-Kyoung Yoon ${ }^{5}$, Heon-Jeong Lee ${ }^{5}$, Leen Kim ${ }^{5}$ and Chang-Hwan Im $^{2 *}$}

\author{
${ }^{1}$ Department of Neurology, Korea University College of Medicine, Seoul, Korea \\ ${ }^{2}$ Department of Biomedical Engineering, Hanyang University, Seoul, Korea \\ ${ }^{3}$ Department of Biomedical Engineering, Yonsei University, Wonju, Korea \\ ${ }^{4}$ BK21 Program for Biomedical Science, Korea University College of Medicine, Seoul, Korea \\ ${ }^{5}$ Department of Psychiatry, Korea University College of Medicine, Seoul, Korea
}

\section{Edited by:}

Mehmet Y. Agargün, Yuzuncu Yil University School of Medicine, Turkey

\section{Reviewed by:}

Mehmet Y. Agargün, Yuzuncu Yil University School of Medicine, Turkey Mitsuyuki Nakao, Tohoku University, Japan

\section{*Correspondence:}

Ki-Young Jung, Department of Neurology, Korea University College of Medicine, \#126-1, Anam-Dong 5Ga, Seongbuk-gu, Seoul 136-705, Korea.

e-mail: jungky@korea.ac.kr; Chang-Hwan Im, Department of Biomedical Engineering, Hanyang University, 17 Haengdang-dong, Seongdong-gu, Seoul 133-791, Korea. e-mail: ich@hanyang.ac.kr
The authors hypothesized that if locomotor drive increases along with rapid eye movement (REM) sleep without atonia in idiopathic REM sleep behavior disorder (RBD), then RBD patients would have greater corticomuscular coherence (CMC) values during REM sleep than at other sleep stages and than in healthy control subjects during REM sleep. To explore this hypothesis, we analyzed beta frequency range $\mathrm{CMC}$ between sensorimotor cortex electroencephalography (EEG) and chin/limb muscle EMG in idiopathic RBD patients. Eleven drug naive idiopathic RBD patients and 11 age-matched healthy control subjects were included in the present study. All participants completed subjective sleep questionnaires and underwent polysomnography for one night. The $\mathrm{CMC}$ value between EEGs recorded at central electrodes and EMGs acquired at leg and chin muscles were computed and compared by repeated measures analysis of variance (ANOVA). Sleep stages and muscle (i.e., chin vs. leg) served as within-subject factors, and group served as the between-subject factor. Repeated measures ANOVA revealed no significant main effect of group $\left(F_{1,20}=0.571, p=0.458\right)$ or muscle $\left(F_{1,20}=1.283, p=0.271\right)$. However, sleep stage was found to have a significant main effect $\left(F_{2.067,41.332}=20.912, p<0.001\right)$. The interaction between group and sleep stage was significant $\left(F_{2.067,41.332}=3.438, p=0.040\right)$. RBD patients had a significantly higher CMC value than controls during REM sleep $(0.047 \pm 0.00$ vs. $0.052 \pm 0.00$, respectively, $p=0.007)$. This study reveals increased CMC during REM sleep in patients with RBD, which indicates increased cortical locomotor drive. Furthermore, this study supports the hypothesis that sufficient locomotor drive plays a role in the pathophysiology of RBD in addition to REM sleep without atonia.

Keywords: REM sleep behavior disorder, pathophysiology, corticomuscular coherence, REM sleep without atonia

\section{INTRODUCTION}

Rapid eye movement (REM) sleep behavior disorder (RBD) is a parasomnia characterized by dream enactment and lack of normal atonia during REM sleep (Ju et al., 2011). Polysomnographic (PSG) recording reveals intermittent or complete loss of REM sleep muscle atonia and excessive phasic and tonic electromyography (EMG) activity during REM sleep in these patients (Fantini et al., 2005; Postuma et al., 2010). It has been proposed that the sublaterodorsal or analogous nucleus projects directly to spinal interneurons, causing active inhibition of muscle activity during REM sleep (Frenette, 2010). The structure in man analogous to the subcoeruleus region in cat and the sublaterodorsal nucleus in rat has been proposed as the nucleus crucial to the pathophysiology of RBD (Boeve et al, 2007; Luppi et al., 2010). Lesion, degeneration, or pharmacological manipulations of the sublaterodorsal magnocellular reticular formation are sufficient to cause REM sleep without atonia (RSWA), but insufficient to cause dream enactment behavior (DEB; Mahowald and Schenck, 2009). Thus, sufficient locomotor drive is necessary in the setting of RSWA to result in clinical
RBD on a nightly basis (Boeve et al., 2007; Luppi et al., 2010). The locomotor generators, which are presumed to project to the spinal motoneurons directly or indirectly via other brainstem nuclei, have yet to be identified and characterized (MacKayLyons, 2002). However, supraspinal inputs play a major role in initiating locomotion, and the sensorimotor cortex is one of main supraspinal centers for spinal locomotor generation (MacKay-Lyons, 2002).

It has been well documented that oscillatory activity of the sensorimotor cortex, measured by electroencephalography (EEG) or magnetoencephalography (MEG), shows coherence in beta frequency range with the results of EMG during weak to moderate steady contractions in both animals and humans. This corticomuscular coherence (CMC) is regarded as efferent phenomenon, i.e., oscillations are propagated from the cortical source to spinal motoneurons via the corticospinal tract (Ushiyama et al., 2010). Furthermore, because CMC quantitatively reflects the corticomotoneuronal coupling, it can be used as an index of the cortical locomotor drive to a particular motor output (Mima and Hallett, 1999). 
We hypothesized that if locomotor drive increases along with RSWA in idiopathic RBD, then RBD patients would have greater beta frequency CMC values during REM sleep than at other sleep stages and than in healthy control subjects during REM sleep. To examine this hypothesis, we analyzed beta frequency range CMC between sensorimotor cortex EEG and chin/limb muscle EMG obtained from PSG data in RBD patients. To the best of our knowledge, this is the first study to address locomotor drive in RBD.

\section{MATERIALS AND METHODS SUBJECTS}

Eleven drug naïve RBD patients were included in the present study. All patients underwent a standardized interview using a structured sleep questionnaire, clinical neurological examinations, and single overnight PSG. The diagnosis of RBD was made based on both clinical and video-PSG as described by the second edition of the International Classification of Sleep Disorders (American Academy of Sleep Medicine, 2005; Iber and American Academy of Sleep Medicine, 2007). All patients had a typical history of DEB, as described by their spouses or family members. PSG documented RSWA in all patients (see below).

The exclusion criteria applied were; the use of medications known to affect REM sleep or tonic or phasic muscle activity (e.g., clonazepam or serotonin reuptake inhibitors), and the presence of another sleep disorder, such as, narcolepsy or moderate to severe sleep apnea (AHI > 15). RBD severities were determined using the RBD severity scale (RBDSS); a simple video-PSG rating scale, proposed by Sixel-Doring et al. (2011). Eleven healthy volunteers were recruited by advertising in the local community. All control subjects completed a detailed clinical interview and a sleep questionnaire, and subsequently, those without any significant sleep problem including RBD underwent overnight PSG. Control subjects had no history of any neurologic, psychiatric, or systemic illness and no family history of a neurodegenerative disorder. All subjects provided written informed consent, and the experimental protocol was approved by the Institutional Review Board of the Korea University Medical Center.

\section{POLYSOMNOGRAPHIC RECORDINGS}

All subjects underwent one night of PSG recording in our sleep laboratory. Sleep studies were recorded using a Somnologica (Embla, Broomfield, CO, USA). Overnight PSG was performed using a six-channel electroencephalography (EEG, F3/A2; F4/A1; C3/A2; C4/A1; O1/A2; O2/A1), a two-channel electrooculogram (EOG), an EMG (of submental, intercostal, and anterior tibialis muscles), and an electrocardiogram with surface electrodes. In order to avoid the aliasing effects, an anti-aliasing filter with a bandwidth of $70 \mathrm{~Hz}$ was employed before the EEG and EMG signals were sampled at $200 \mathrm{~Hz}$.

A thermistor (for monitoring nasal airflow), a nasal air pressure monitor, an oximeter (for measuring oxygen saturation), piezoelectric bands (for determining thoracic and abdominal wall motion), and a body position sensor were also attached. Patients were recorded on videotape using an infrared video camera and were continuously observed by a PSG technician.

Sleep architecture was scored in 30-s epochs, and sleep staging was performed according to the standard criteria described by
Rechtschaffen and Kales (1968). RSWA was scored as described in the AASM manual for the scoring of sleep and associated events (Iber and American Academy of Sleep Medicine, 2007), which include sustained muscle activity or excessive transient muscle activity. Sustained muscle activity during REM sleep was defined as REM sleep demonstrating a chin EMG amplitude greater than the minimum amplitude during non-REM sleep of duration $>50 \%$ the duration of the 30-s epoch. Excessive transient muscle activity during REM sleep was defined to be present when $>50 \%$ of $30-\mathrm{s}$ epoch contained transient muscle activity bursts of amplitude $>4$ times that of background EMG activity.

\section{ATONIA INDEX}

The atonia index was devised by Ferri et al. (2008) to quantify the amplitude of submentalis muscle EMG during sleep, and has been used for the diagnosis of RBD. To calculate atonia index values, the rectified chin EMG signal was subdivided into 1-s miniepochs, and the average amplitude of each of these was calculated. Then, we applied a correction method which subtracted from each mini-epoch average rectified EMG amplitude, the minimum value found in a moving window including the 60 mini-epochs surrounding it (Ferri et al., 2010).

Atonia index was defined as the ratio between the percentage of EMG mini-epochs with an average amplitude of $\leq 1 \mu \mathrm{V}$ and the total mini-epochs excluding those with $1 \mu \mathrm{V}<$ averaged EMG amplitude $\leq 2 \mu \mathrm{V}$. Possible atonia index values range from 0 to 1 , where values close to 0 indicate the absence of EMG atonia and values close to 1 indicate stable EMG atonia. This index was calculated for each sleep stage.

\section{CORTICOMUSCULAR COHERENCE}

Coherence is an extension of Pearson's correlation coefficient in the frequency domain and is defined as the cross-spectral density normalized by auto-spectra. The coherence $C_{x y}$ between two signals $x$ and $y$ can be calculated using;

$$
C_{x y}(f)=\frac{\left|P_{x y}(f)\right|^{2}}{P_{x x}(f) P_{y y}(f)},
$$

where $f$ represents a frequency of interest, $P_{x x}$ and $P_{y y}$ are the autospectra of $x$ and $y$, respectively, and $P_{x y}$ is the cross-spectral density of the two signals. This procedure returns a real number between 0 (no coherence) and 1 (perfect coherence).

In the present study, CMC values between EEGs recorded at central electrodes (C3 and C4) and EMGs acquired at leg (pretibial) and chin (submental) muscles were computed using NeuroSpec 2.0 toolbox (www.neurospec.org) implemented in MATLAB 7.1 (MathWorks Inc., USA).

Four CMC values (C3 EEG - chin EMG, C4 EEG - chin EMG, C3 EEG - right leg EMG, C4 EEG - left leg EMG) were computed for each 30-s epoch (6,000 time samples per epoch) based on the fast Fourier transform (FFT) analysis for 23 non-overlapping windows with 256 time samples. This gave a frequency resolution of approximately $0.78 \mathrm{~Hz}$. To avoid influence of muscle activity during DEB on coherence measure, we excluded epochs being contaminated by sufficient amount of muscle and movement artifacts during DEB, which were identified by video monitoring, 
from coherence analysis. However, epochs containing either phasic EMG activity due to REM or RSWA not accompanied by DEB were included in the present study.

Before evaluating CMC values, EMG signals were rectified so as to enhance the firing rate information (Myers et al., 2003). Since beta band CMC is known to be most closely associated with motor activities (Conway et al., 1995; Halliday et al., 1998; Mima and Hallett, 1999), CMC values in beta frequency band $(12-30 \mathrm{~Hz})$ were averaged to obtain a single CMC value for each sleep stage.

\section{STATISTICAL ANALYSIS}

Statistical analysis was performed using SPSS software (Version 10, SPSS, Inc.). The Mann-Whitney $U$ test was used to compare of clinical and PSG variables between groups, and repeated measures analysis of variance (ANOVA) was used to analyze CMC and atonia index. Sleep stage (five levels: W, R, N1, N2, and N3) and muscle (two levels: chin and leg EMG) served as within-subject factors, and group (i.e., RBD patients vs. Controls) served as the between-subject factor. The Greenhouse-Geisser correction was used to evaluate $F$ ratios to control for Type 1 error in the repeated measures design. Bonferroni's post hoc tests were used to identify the sources found significant by ANOVA. Spearman's rank correlation analysis was performed to evaluate correlations between $\mathrm{CMC}$ and clinical variables, that is, age of onset, disease duration, RBD frequency, RBDSS, and atonia index. $p$ Values of $<0.05$ were considered significant.

\section{RESULTS}

\section{CLINICAL AND POLYSOMNOGRAPHIC CHARACTERISTICS}

Mean patient age was $63.8 \pm 9.0$ years (range, $44-73$ years) and mean disease duration was $3.3 \pm 2.3$ years (range, $1-8$ years). The frequency of RBD episodes ranged from daily to weekly in all patients (Table 1). Neurologic examinations were unremarkable in all patients. All patients showed either the presence of excessive tonic chin EMG activity during REM sleep, or excessive phasic submental or limb EMG twitching by overnight PSG. Mean RBDSS was $3.1 \pm 0.9$ (range, 2-4). Patients and controls were well-matched for age, sex, and BMI. Subjective sleep quality (PSQI and AIS) and excessive daytime sleepiness (defined by ESS $>10$ ) were not significantly different between patients and controls (Table 1). PSG revealed that RBD patients showed poorer sleep efficiency and a lower percentage of N2 sleep than controls (Table 2). Control subjects had a higher mean apnea-hypopnea index compared to RBD patients ( $7.5 \pm 5.4$ vs. $2.3 \pm 2.6$, respectively; $p=0.011)$. None of the patients had an AHI $>10$ whereas four of the controls did.

\section{ATONIA INDEX}

Repeated measures ANOVA revealed no significant main effect for group. However, sleep stage was found to have a significant effect $\left(F_{2.6,51.4}=24.528, p<0.001\right)$. The interaction between group and sleep stage was also significant $\left(F_{2.6,51.4}=3.979, p=0.017\right.$, Figure 1). In control subjects, the atonia index of the waking stage was significantly lower than for other sleep stages. Contrary to this, in patients, the atonia index of the waking stage was significantly lower than those of the $\mathrm{N} 2$ and $\mathrm{N} 3$ sleep only. REM sleep atonia index was similar to atonia index of waking stage in patients. RBD patients had a significantly lower mean atonia
Table 1 | Patient demographic and subjective sleep characteristics.

\begin{tabular}{llll}
\hline & RBD & Control & $\boldsymbol{p}$ Value \\
\hline$N$ & 11 & 11 & \\
Sex & M8F3 & M7F4 & 0.647 \\
Age (years) & $62.9 \pm 10.4$ & $60.5 \pm 8.3$ & 0.548 \\
BMI $\left(\mathrm{kg} / \mathrm{m}^{2}\right)$ & $24.5 \pm 2.5$ & $23.2 \pm 2.6$ & 0.239 \\
PSQI & $6.9 \pm 3.3$ & $5.0 \pm 2.7$ & 0.161 \\
ESS & $5.7 \pm 3.7$ & $4.4 \pm 2.5$ & 0.334 \\
AIS & $6.1 \pm 2.9$ & $4.2 \pm 3.0$ & 0.187 \\
\hline
\end{tabular}

BMI, body mass index; PSQI, Pittsburgh Sleep Quality Index; ESS, Epworth Sleepiness Scale; AIS, Athens Insomnia Scale.

Table 2 | Polysomnographic variables.

\begin{tabular}{llll}
\hline & RBD & Control & $\boldsymbol{p}$ Value \\
\hline TIB (min) & $439.3 \pm 36.6$ & $426.1 \pm 48.5$ & 0.478 \\
TST (min) & $321.4 \pm 82.4$ & $371.4 \pm 40.8$ & 0.086 \\
WASO (min) & $69.5 \pm 34.2$ & $43.4 \pm 37.4$ & 0.062 \\
SE (\%) & $73.7 \pm 18.9$ & $87.6 \pm 7.7$ & 0.035 \\
SL (min) & $37.1 \pm 75.5$ & $11.3 \pm 18.1$ & 0.284 \\
N1 (\%) & $23.09 \pm 12.0$ & $24.2 \pm 6.6$ & 0.777 \\
N2 (\%) & $41.9 \pm 3.7$ & $46.9 \pm 7.0$ & 0.048 \\
N3 (\%) & $10.7 \pm 10.0$ & $9.1 \pm 7.0$ & 0.673 \\
$R(\%)$ & $21.0 \pm 7.6$ & $19.7 \pm 4.7$ & 0.637 \\
AI (\#/h) & $27.2 \pm 11.8$ & $26.5 \pm 6.8$ & 0.864 \\
AHI (\#/h) & $2.3 \pm 2.6$ & $7.5 \pm 5.4$ & 0.011 \\
PLMSI (\#/h) & $23.9 \pm 28.3$ & $12.1 \pm 33.3$ & 0.383 \\
\hline
\end{tabular}

TIB, time in bed; TST, total sleep time; WASO, wake time after sleep onset; SE, sleep efficiency; SL, sleep latency; Al: arousal index, AHI: apnea-hypopnea index; $P L M S I$, periodic leg movement during sleep index.

index than controls during REM sleep $(0.76 \pm 0.18$ vs. $0.97 \pm 0.02$, respectively, $p=0.001)$. atonia index was found to be marginally correlated with RBD severity in RBD patients $(r=-0.558$, $p=0.057)$. Age of onset was not significantly correlated with REM sleep atonia index $(r=-0.642, p=0.033)$.

\section{CORTICOMUSCULAR COHERENCE}

Figure 2 shows an overnight leg-CMC plot for a control subject (A) and an RBD patient (B). Repeated measures ANOVA revealed no significant main effect of group $\left(F_{1,20}=0.571, p=0.458\right)$ or muscle $\left(F_{1,20}=1.283, p=0.271\right)$. However, sleep stage was found to have a significant main effect $\left(F_{2.067,41.332}=20.092, p<0.001\right)$. The interaction between group and sleep stage was significant $\left(F_{2.067,41.332}=3.438, p=0.040\right.$; Figure 3).

In controls, $\mathrm{CMC}$ of waking stage was significantly higher than those of other sleep stages. In other words, CMC during REM sleep was significantly lower compared to waking stage. Contrary to this, in patients, CMC during N3 sleep was significantly lower than those of other sleep stages. REM sleep CMC value was not significantly different from that of waking stage in patients. RBD patients had a significantly higher CMC value than controls during REM sleep $(0.047 \pm 0.00$ vs. $0.052 \pm 0.00$, respectively, $p=0.007)$. 


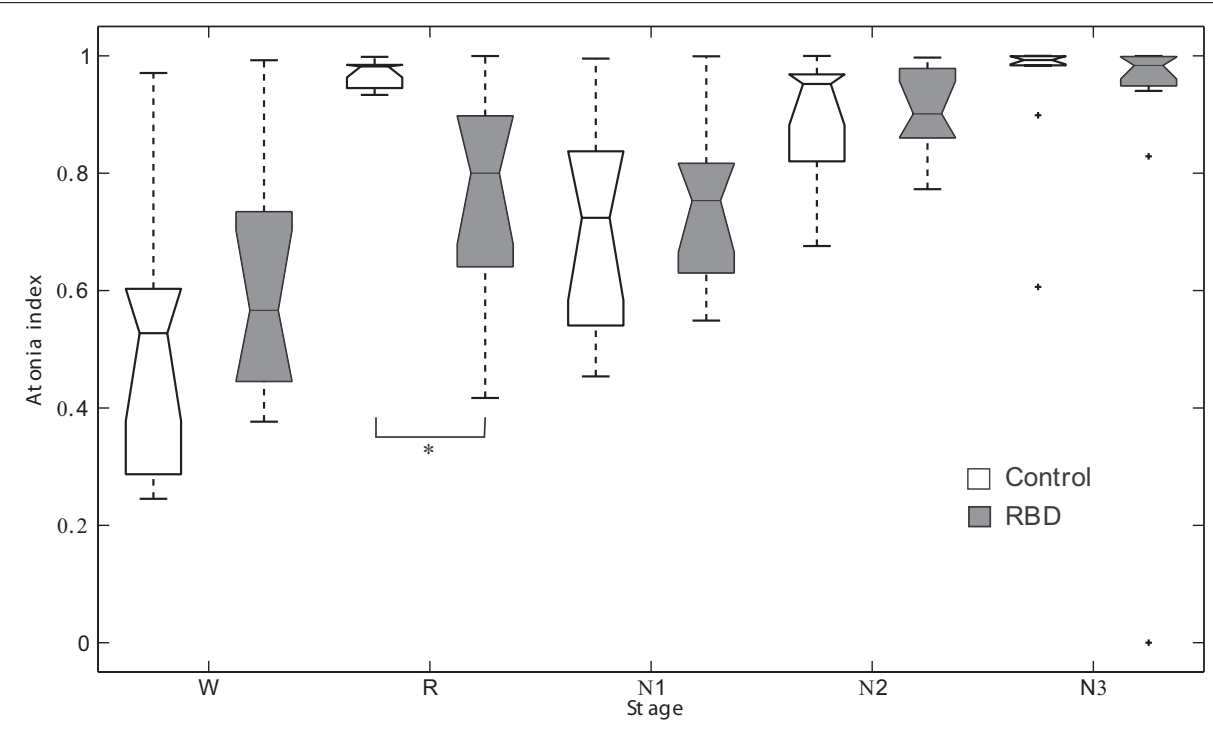

FIGURE 1 | Box plots of the atonia index of each sleep stage. *Denotes $p<0.001$. RBD, REM sleep behavior disorder; W, wakefulness; R, REM sleep stage; N1, non-REM sleep stage 1; N2, non-REM sleep stage 2; N3, non-REM sleep stage 3.
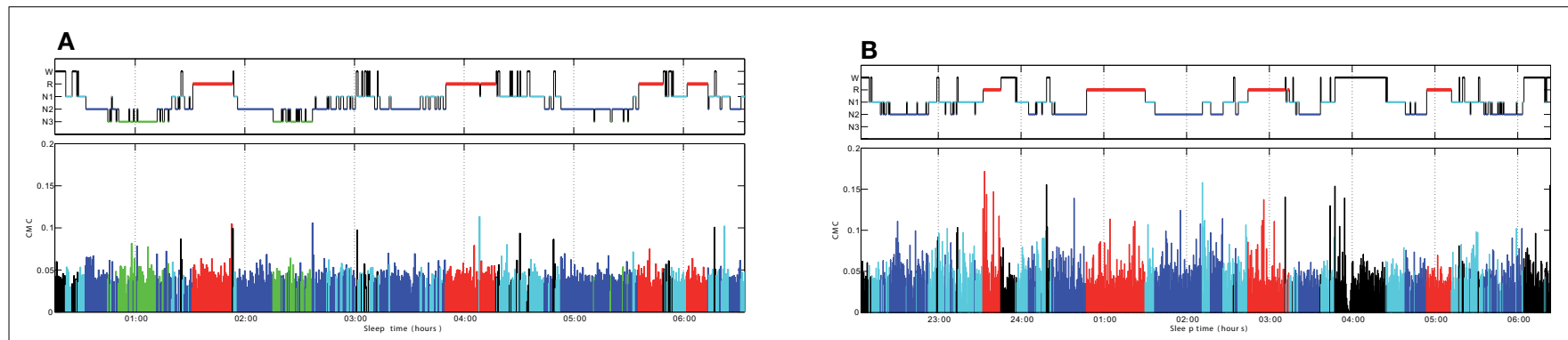

FIGURE 2 | Overnight corticomuscular coherence for a control subject (A) and an RBD patient (B). CMC, corticomuscular coherence; W, wakefulness; $\mathrm{R}$, REM sleep stage; N1, non-REM sleep stage 1; N2, non-REM sleep stage 2; N3, non-REM sleep stage 3.

\section{CORRELATION BETWEEN CMC AND CLINICAL VARIABLES}

Corticomuscular coherence during REM sleep showed significant correlation with clinical variables. However, other sleep stages did not show noteworthy correlation between CMC and clinical variables. A significant negative correlation was observed between REM sleep CMC and age of RBD onset $(r=-0.642$, $p=0.033)$. RBDSS tended to modestly correlated with REM sleep CMC $(r=0.575, p=0.064)$. However, disease duration, severity, and frequency of RBD were not found to be correlated with REM sleep CMC. On the other hand, atonia index was found to be significantly correlated with REM sleep CMC $(r=-0.662$, $p=0.027)$.

\section{DISCUSSION}

Rapid eye movement sleep behavior disorder is characterized by vigorous DEB and abnormally increased phasic and/or tonic EMG activity during REM sleep (Iranzo et al., 2009b). Because RSWA is insufficient to cause DEB, a core symptom of RBD, increased locomotor drive and/or RSWA has been suggested to be responsible for the clinical expression of human RBD (Boeve et al., 2007). RSWA has been extensively studied in animal and human experiments.
However, unlike RSWA, no report has been issued to date regarding locomotor drive in RBD.

To investigate locomotor drive during REM sleep, we compared the beta frequency CMC values of RBD patients and normal controls. After carefully removing epochs of $\mathrm{DEB}$, it was found in the present study that CMC during REM sleep in RBD patients was significantly higher than in controls, but that other sleep stages were no different. Coherent oscillations of neurons in the sensorimotor cortex have been shown to be involved in the corticospinal control of muscle activity (Baker, 2007; Witham et al., 2010), and this interaction between sensorimotor cortex and muscle has been measured by analysis of CMC in the beta frequency range (Conway et al., 1995). Furthermore, it has been widely assumed that beta frequency CMC reflects direct coupling between cortex and muscles, and hence, our results indicate that increased corticomotoneuronal coupling is present in RBD.

Although the locomotor generators have yet to be identified and characterized, supratentorial influences on both locomotor generators and the muscle atonia system are suggested (Boeve, 2010). Increased phasic EMG activity is a characteristic of RBD (Iranzo et al., 2009a). Although the origin of phasic EMG activity 


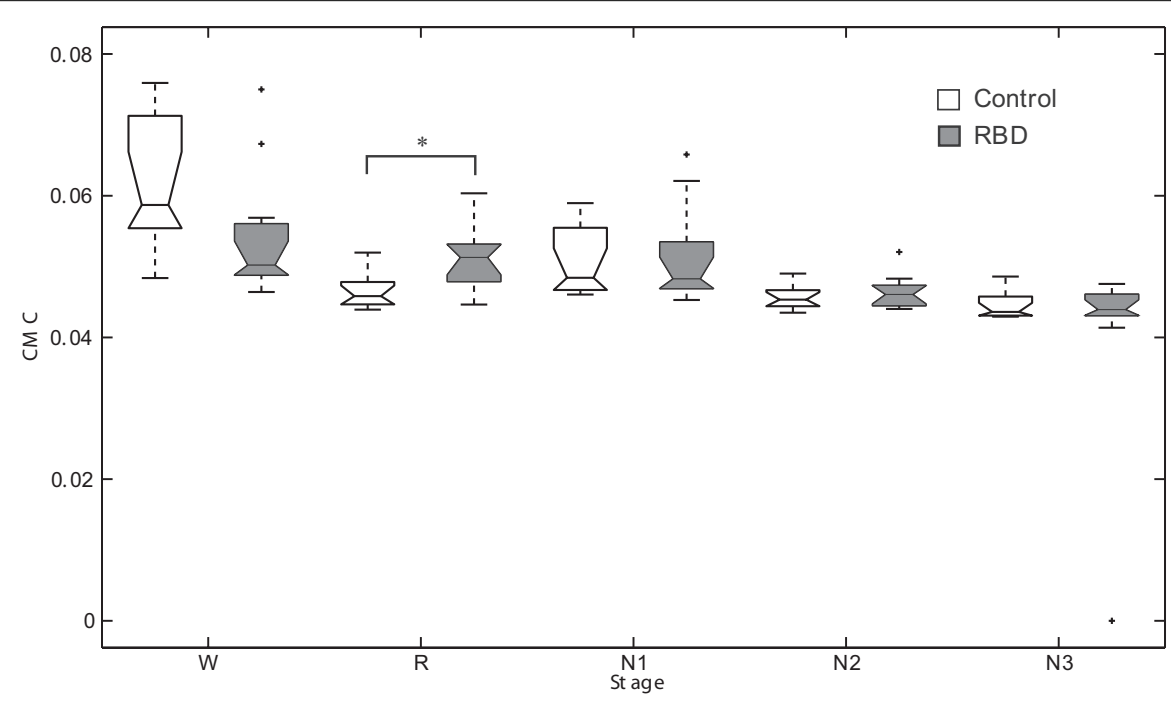

FIGURE 3 | Box plots of corticomuscular coherence of each sleep stage. *Denotes $p<0.05$. RBD, REM sleep behavior disorder; W, wakefulness; R, REM sleep stage; N1, non-REM sleep stage 1; N2, non-REM sleep stage 2; N3, non-REM sleep stage 3.

during REM sleep is unknown, it is postulated that it is due to the activation of motor pathways arising from the pyramidal cells of the motor cortex directly projecting to motoneurons or indirectly projecting via pre-motoneurons located in pontine and medullary reticular nuclei and intermediate spinal cord (Luppi et al., 2010). Thus, increased corticomuscular coupling during REM sleep may indicate that cortical projection to motor neurons is a source of locomotor drive during REM sleep. In addition, it has been reported that cortical signals precede motor unit firing and appear to reflect modulation of the common central drive to the spinal motoneuron pool (Hari and Salenius, 1999; Witham et al., 2010).

Taken together, our findings suggest that increased cortical locomotor drive during REM sleep is present in RBD. Accordingly, our study supports the hypothesis that sufficient locomotor drive may play a role in the pathophysiology of RBD, particularly causing DEB. Recently, increased cerebral perfusion in the supplementary motor area was reported during DEB in an RBD patient (Yves et al., 2011), and it was suggested that the supplementary motor area plays a role in the generation of $\mathrm{DEB}$ in $\mathrm{RBD}$, which concurs with our findings.

It has been suggested that CMC is mediated by both ascending and descending pathways. Proprioceptive feedback is known to play a role in the beta-range CMC. Oscillations may hold overt motor output constant in order to render the interpretation of the proprioceptive state more effective (Baker, 2007). Thus, it is possible that increased phasic/tonic muscle activity may contribute in part to increase proprioceptive afferent signal to EEG, hence increased beta CMC in RBD patients.

Evidence indicates that the cerebral cortex is involved in the pathogenesis of idiopathic RBD. EEG studies have shown that idiopathic RBD patients showed impaired cortical activity during both REM sleep and wakefulness (Fantini et al., 2003; MassicotteMarquez et al., 2005; Iranzo et al., 2010). Furthermore, diffusion tensor imaging and voxel based morphometry of brain MRI studies have shown multiple cortical abnormalities, such as, in olfactory regions, in the temporal lobe, including the hippocampus and parahippocampal gyri, and in visual cortices (Unger et al., 2010; Scherfler et al., 2011). A PET study revealed hypometabolism in multiple cortical regions including the anterior cingulate gyrus, and frontal, anterior temporal, and occipital lobes in idiopathic RBD patients (Fujishiro et al., 2010). In addition, brain SPECT demonstrated reduced regional cerebral blood flow in the frontal and temporoparietal lobes in patients with idiopathic RBD (Mazza et al., 2006). Therefore, cortical mechanism in addition to a brainstem mechanism may be involved in the pathogenesis of RBD.

Atonia index was introduced to measure quantitatively EMG atonia during sleep (Ferri et al., 2008). In the present study, REM sleep atonia index of RBD patients was significantly lower than that of control subjects, confirming previous reports (Ferri et al., $2008,2010)$. CMC was found to be significantly correlated with atonia index and marginally correlated with RBD severity. This suggests that REM sleep CMC might be used as quantitative index of RBD. REM sleep CMC was also significantly correlated with onset age of RBD, although disease duration did not show any relationship with REM sleep CMC. Considering progressive nature of RBD (Iranzo et al., 2009b), our result indicates that $\mathrm{RBD}$ with earlier onset, which have higher $\mathrm{CMC}$, may progress faster than those with later onset during the course of disease process.

Summarizing, this study shows for the first time, increased CMC during REM sleep in patients with RBD, which indicates an increased cortical locomotor drive. Furthermore, this study supports the hypothesis that sufficient locomotor drive plays a role in the pathophysiology of RBD in addition to RSWA.

\section{ACKNOWLEDGMENTS}

This work was supported by the National Research Foundation of Korea (NRF) grant funded by the Korea government (MEST) (No. 2011-0029740) and in part by grant from Korea University (K1127171) and by the research fund of Hanyang University (HY-2011-N). 


\section{REFERENCES}

American Academy of Sleep Medicine. (2005). The International Classification of Sleep Disorders: Diagnostic and Coding Manual, 2nd Edn. Westchester, IL: American Academy of Sleep Medicine.

Baker, S. N. (2007). Oscillatory interactions between sensorimotor cortex and the periphery. Curr. Opin. Neurobiol. 17, 649-655.

Boeve, B. F. (2010). REM sleep behavior disorder: updated review of the core features, the REM sleep behavior disorder-neurodegenerative disease association, evolving concepts, controversies, and future directions. Ann. N. Y. Acad. Sci. 1184, 15-54.

Boeve, B. F., Silber, M. H., Saper, C. B., Ferman, T. J., Dickson, D. W., Parisi, J. E., Benarroch, E. E., Ahlsko, J. E., Smith, G. E., Caselli, R. C., TippmanPeikert, M., Olson, E. J., Lin, S. C., Young, T., Wszolek, Z., Schenck, C. H., Mahowald, M. W., Castillo, P. R., Del Tredici, K., and Braak, H. (2007). Pathophysiology of REM sleep behaviour disorder and relevance to neurodegenerative disease. Brain 130(Pt 11), 2770-2788.

Conway, B. A., Halliday, D. M., Farmer, S. F., Shahani, U., Maas, P., Weir, A. I., and Rosenberg, J. R. (1995). Synchronization between motor cortex and spinal motoneuronal pool during the performance of a maintained motor task in man. J. Physiol. (Lond.) 489(Pt 3), 917-924.

Fantini, M. L., Ferini-Strambi, L., and Montplaisir, J. (2005). Idiopathic REM sleep behavior disorder: toward a better nosologic definition. Neurology 64, 780-786.

Fantini, M. L., Gagnon, J. F., Petit, D., Rompre, S., Decary, A., Carrier, J., and Montplaisir, J. (2003). Slowing of electroencephalogram in rapid eye movement sleep behavior disorder. Ann. Neurol. 53, 774-780.

Ferri, R., Manconi, M., Plazzi, G., Bruni, O., Vandi, S., Montagna, P., FeriniStrambi, L., and Zucconi, M. (2008). A quantitative statistical analysis of the submentalis muscle EMG amplitude during sleep in normal controls and patients with REM sleep behavior disorder. J. Sleep Res. 17, 89-100.

Ferri, R., Rundo, F., Manconi, M., Plazzi, G., Bruni, O., Oldani, A., Ferini-Strambi, L., and Zucconi, M. (2010). Improved computation of the atonia index in normal controls and patients with REM sleep behavior disorder. Sleep Med. 11, 947-949.

Frenette, E. (2010). REM sleep behavior disorder. Med. Clin. North Am. 94, 593-614.

Fujishiro, H., Iseki, E., Murayama, N., Yamamoto, R., Higashi, S., Kasanuki, K., Suzuki, M., Arai, H., and Sato, K. (2010). Diffuse occipital hypometabolism on [18 F]-Fdg pet scans in patients with idiopathic REM sleep behavior disorder: prodromal dementia with Lewy bodies? Psychogeriatrics 10, 144-152.

Halliday, D. M., Conway, B. A., Farmer, S. F., and Rosenberg, J. R. (1998). Using electroencephalography to study functional coupling between cortical activity and electromyograms during voluntary contractions in humans. Neurosci. Lett. 241, 5-8.

Hari, R., and Salenius, S. (1999). Rhythmical corticomotor communication. Neuroreport 10, R1-R10.

Iber, C., and American Academy of Sleep Medicine. (2007). The AASM Manual for the Scoring of Sleep and Associated Events: Rules, Terminology and Technical Specifications. Westchester, IL: American Academy of Sleep Medicine.

Iranzo, A., Isetta, V., Molinuevo, J. L., Serradell, M., Navajas, D., Farre, R., and Santamaria, J. (2010). Electroencephalographic slowing heralds mild cognitive impairment in idiopathic REM sleep behavior disorder. Sleep Med. 11, 534-539.

Iranzo, A., Ratti, P. L., Casanova-Molla, J., Serradell, M., Vilaseca, I., and Santamaria, J. (2009a). Excessive muscle activity increases over time in idiopathic REM sleep behavior disorder. Sleep 32, 1149-1153.

Iranzo, A., Santamaria, J., and Tolosa, E. (2009b). The clinical and pathophysiological relevance of REM sleep behavior disorder in neurodegenerative diseases. Sleep Med. Rev. 13, 385-401.

Ju, Y. E., Larson-Prior, L., and Duntley, S. (2011). Changing demographics in REM sleep behavior disorder: possible effect of autoimmunity and antidepressants. Sleep Med. 12, 278-283.

Luppi, P. H., Clement, O., Sapin, E., Gervasoni, D., Peyron, C., Leger, L., Salvert, D., and Fort, P. (2010). The neuronal network responsible for paradoxical sleep and its dysfunctions causing narcolepsy and rapid eye movement (REM) behavior disorder. Sleep Med. Rev. 15, 153 163.

MacKay-Lyons, M. (2002). Central pattern generation of locomotion: a review of the evidence. Phys. Ther. 82, 69-83.

Mahowald, M. W., and Schenck, C. H. (2009). The REM sleep behavior disorder odyssey. Sleep Med. Rev. 13, 381-384.

Massicotte-Marquez, J., Carrier, J., Decary, A., Mathieu, A., Vendette, M., Petit, D., and Montplaisir, J. (2005). Slow-wave sleep and delta power in rapid eye movement sleep behavior disorder. Ann. Neurol. 57, 277-282.

Mazza, S., Soucy, J. P., Gravel, P., Michaud, M., Postuma, R., Massicotte-Marquez, J., Decary, A., and Montplaisir, J. (2006). Assessing whole brain perfusion changes in patients with REM sleep behavior disorder. Neurology 67, 1618-1622.

Mima, T., and Hallett, M. (1999). Corticomuscular coherence: a review. J. Clin. Neurophysiol. 16, 501-511.

Myers, L. J., Lowery, M., O’Malley, M., Vaughan, C. L., Heneghan, C., St Clair Gibson, A., Harley, Y. X., and Sreenivasan, R. (2003). Rectification and non-linear pre-processing of EMG signals for cortico-muscular analysis. J. Neurosci. Methods 124, 157-165.

Postuma, R. B., Gagnon, J. F., Rompre, S., and Montplaisir, J. Y. (2010). Severity of REM atonia loss in idiopathic REM sleep behavior disorder predicts Parkinson disease. Neurology 74, 239-244.

Rechtschaffen, A., and Kales A., eds. (1968). A Manual of Standardized Terminology, Technique and Scoring System for Sleep Stages of Human Subjects. Los Angeles: Brain Information Service/Brain Research Institute, UCLA.

Scherfler, C., Frauscher, B., Schocke, M., Iranzo, A., Gschliesser, V., Seppi, K., Santamaria, J., Tolosa, E., Högl, B., Poewe, W., and (SINBAR )(Sleep Innsbruck Barcelona) Group. (2011). White and gray matter abnormalities in idiopathic rapid eye movement sleep behavior disorder: a diffusion-tensor imaging and voxel-based morphometry study. Ann. Neurol. 69, 400-407.

Sixel-Doring, F., Schweitzer, M., Mollenhauer, B., and Trenkwalder, C. (2011). Intraindividual variability of REM sleep behavior disorder in Parkinson's disease: a comparative assessment using a new REM sleep behavior disorder severity scale (RBDSS) for clinical routine. J. Clin. Sleep Med. 7, 75-80.

Unger, M. M., Belke, M., Menzler, K., Heverhagen, J. T., Keil, B., Stiasny-Kolster, K., Rosenow, F., Diederich, N. J., Mayer, G., Möller, J. C., Oertel, W. H., and Knake, S. (2010). Diffusion tensor imaging in idiopathic REM sleep behavior disorder reveals microstructural changes in the brainstem, substantia nigra, olfactory region, and other brain regions. Sleep 33, 767-773.

Ushiyama, J., Takahashi, Y., and Ushiba, J. (2010). Muscle dependency of corticomuscular coherence in upper and lower limb muscles and training-related alterations in ballet dancers and weightlifters. J. Appl. Physiol. 109, 1086-1095.

Witham, C. L., Wang, M., and Baker, S. N. (2010). Corticomuscular coherence between motor cortex, somatosensory areas and forearm muscles in the monkey. Front. Syst. Neurosci. 4:38. doi:10.3389/fnsys.2010.00038

Yves, D., Vincent, B., Regis, L., Audrey, G., Valerie, C. D., Sophie, B., and Philippe, P. (2011) Increased perfusion in supplementary motor area during a REM sleep behaviour episode. Sleep Med. 12, 531-532.

Conflict of Interest Statement: The authors declare that the research was conducted in the absence of any commercial or financial relationships that could be construed as a potential conflict of interest.

Received: 02 November 2011; accepted: 28 March 2012; published online: 23 April 2012.

Citation: Jung K-Y, Cho J-H, Ko D, Seok $H Y$, Yoon $H-K$, Lee H-J, Kim $L$ and Im C-H (2012) Increased corticomuscular coherence in idiopathic REM sleep behavior disorder. Front. Neur. 3:60. doi: 10.3389/fneur.2012.00060

This article was submitted to Frontiers in Sleep and Chronobiology, a specialty of Frontiers in Neurology.

Copyright $\odot 2012$ Jung, Cho, Ko, Seok, Yoon, Lee, Kim and Im. This is an open-access article distributed under the terms of the Creative Commons Attribution Non Commercial License, which permits non-commercial use, distribution, and reproduction in other forums, provided the original authors and source are credited. 